\title{
Adaptação de Instrumentos Neuropsicológicos Verbais: Um Fluxograma de Procedimentos para Além da Tradução
}

\author{
Rochele Paz Fonseca \\ Fabiola Schwengber Casarin \\ Camila Rosa de Oliveira \\ Gigiane Gindri \\ Pontifícia Universidade Católica do Rio Grande do Sul \\ Ellen Cristina Siqueira Soares Ishigaki \\ Karin Zazo Ortiz. \\ Universidade Federal de São Paulo \\ Maria Alice de Mattos Pimenta Parente \\ Universidade Federal do Rio Grande do Sul
}

Lilian Cristine Scherer

Universidade de Santa Cruz do Sul

\begin{abstract}
RESUMO
O uso de versões apenas traduzidas de instrumentos neuropsicológicos padronizados internacionais na clínica brasileira ainda é bastante freqüente. Para que uma ferramenta diagnóstica mensure os processos cognitivos a que se propõe a medir, torna-se essencial uma adaptação de suas instruções e de seus estímulos à realidade brasileira lingüística, cultural e social. O presente artigo de revisão teórica tem por objetivo refletir sobre as particularidades da adaptação de instrumentos neuropsicológicos verbais, propondo um fluxograma de procedimentos de adaptação neuropsilinguística que transcenda à mera tradução. Este fluxograma foi derivado da consulta à literatura de avaliação psicológica, avaliação neuropsicológica, avaliação fonoaudiológica e da experiência clínica e científica das autoras na construção e adaptação de ferramentas clínicas de mensuração de desempenho cognitivo por meio de estímulos verbais. Engloba procedimentos específicos envolvendo três grupos de procedimentos gerais: tradução, análise de juízes e estudo piloto. O contato contínuo com os pesquisadores autores do instrumento original é destacado como uma etapa essencial. A continuidade do processo de adaptação com procedimentos neuropsicométricos é brevemente discutida.
\end{abstract}

Palavras-chave: avaliação neuropsicológica; adaptação; testes verbais; linguagem; neuropsicolinguística.

\section{ABSTRACT \\ Adaptation of Neuropsychological Verbal Instruments: A Fluxogram of Procedures far Beyond Translation}

A very recurrent practice in Brazilian clinics still nowadays is the adoption of translated versions of neuropsychological instruments internationally standardized. It is fundamental that diagnostic tools have their instructions and stimuli adapted to the Brazilian linguistic, cultural and social reality, so that they accurately and reliably measure the cognitive processes that they are meant to assess. This theoretical review article aims to reflect on the specificities underlying the adaptation of verbal neuropsychological instruments, proposing a fluxogram of procedures for neuropsycholinguistic adaptation which transcends mere translation. This fluxogram was derived from the literature on psychological, neuropsychological, phonoaudiological assessments, and from the authors' clinical and scientific experience in the construction and adaptation of tools for the evaluation of cognitive performance by means of verbal stimuli. It encompasses specific procedures comprising three groups of general procedures: translation, judges' analysis and pilot study. The continuous contact with the researchers who are the authors of the original instrument is highlighted as representing an essential phase. The ongoing adaptation process with neuropsychometric procedures is briefly discussed.

Keywords: neuropsychological assessment; adaptation; verbal tests; language; neuropsycholinguistics. 
Rochele Paz Fonseca, Fabíola Schwengber Casarin, Camila Rosa de Oliveira, Gigiane Gindri, Ellen Cristina Siqueira Soares Ishigaki, Karin Zazo Ortiz, Maria Alice de Mattos Pimenta Parente, \& Lilian Cristine Scherer

A avaliação neuropsicológica é uma das intervenções propostas pela neuropsicologia clínica, abrangendo um conjunto de procedimentos de entrevistas, observação e exame padronizado ou formal e não padronizado e/ou flexível e naturalístico do desempenho cognitivo (Strauss, Sherman, \& Spreen, 2006). Este último pilar de procedimentos caracteriza-se pela administração de testes e tarefas que se propõem a mensurar um ou mais processos cognitivos. No entanto, apesar do significativo avanço da neuropsicologia brasileira na última década, os neuropsicólogos nas diferentes áreas em que a neuropsicologia se aplica ainda se deparam com a escassez de instrumentos adaptados para a realidade sócio-cultural e linguística do Brasil, tanto para fins clínicos quanto de pesquisa. Essa falta de ferramentas padronizadas com versões adequadamente adaptadas é ainda mais prejudicial quando o instrumento envolve estímulos verbais, por apresentarem características próprias do país em cuja população o instrumento será administrado. Há, assim, duas principais categorias de testes: (1) aqueles que examinam a linguagem ou comunicação, em que a linguagem é usada para avaliar a si própria, e (2) aqueles que avaliam outras funções cognitivas por meio da linguagem, isto é, testes em que palavras, sentenças e discurso são veículos para mensurar a memória, a atenção, as funções executivas, por exemplo.

Uma solução para suprir a restrita disponibilidade de instrumentos de avaliação neuropsicológica é a adaptação de testes e de baterias reconhecidas internacionalmente que tenham obtido evidências suficientes de fidedignidade, validade, sensibilidade e especificidade na avaliação para a qual de propõem em seu país de origem. A adaptação de instrumentos já validados em outros países tem como vantagem o intercâmbio de informações entre a comunidade científica, além de menores custos (Guillemin, 1995). Entretanto, tornase importante salientar que esse processo não deve se restringir apenas à tradução de instrumentos, uma vez que a língua e a cultura interferem no processo de avaliação neuropsicológica como um todo, desde a sua aplicação até o fornecimento de respostas pelos pacientes e a interpretação destas pelo examinador (Pérez-Arce, 1999).

Mesmo assim, muitos testes e tarefas com estímulos verbais utilizados para examinar quadros de distúrbios lingüísticos e/ou cognitivos apenas passaram por um processo de tradução. Tal procedimento realizado isoladamente não é suficiente para que um teste construído em uma determinada cultura possa ser utilizado em outros países com a mesma qualidade.
Alguns estudos, como o de Radanovic e Mansur (2002) e o de Villaseñor, Carlos, Curiel, Medrano e Rodriguez (2003), salientam que a adaptação de um instrumento de linguagem necessita passar por um processo específico, cujo objetivo é o de minimizar possíveis erros de interpretações, visto que existem especificidades linguísticas e culturais que limitam a aplicação de algumas provas.

Há evidências quantitativas do aumento de publicações acerca da adaptação de instrumentos revisadas por Casillas e Robins (2005). Segundo estes autores, a quantidade de artigos sobre adaptação de instrumentos aumentou sete vezes do período de 1984-1993 a 19942004. No entanto, um dado mostrou-se preocupante nesta revisão sistemática: há mais do que o dobro de publicações recentes com as palavras-chave "test translation" quando comparado com a quantidade de manuscritos sobre "test adaptation". A tradução parece continuar sendo, ainda, a principal etapa de adaptação.

No mesmo contexto da crescente publicação científica sobre adaptação transcultural de instrumentos neuropsicológicos relatada por Casillas e Robins (2005), observa-se uma maior preocupação com a normatização e validação de instrumentos neuropsicológicos no contexto nacional, preocupação que pode ser exemplificada, inclusive, com publicações nacionais como o livro organizado por Ortiz e cols. (2008). Nesta obra, há diversos capítulos que apresentam estudos acerca de diferentes ferramentas neuropsicológicas de avaliação de uma ou mais habilidades neurocognitivas. No entanto, em geral, parece não haver uma sistematização quanto ao processo de adaptação de testes para a população brasileira. Até onde se sabe não há publicações que mencionem e explicitem detalhadamente quais etapas devem ser seguidas, dificultando a padronização de um método a ser usado por neuropsicólogos autores de versões adaptadas de testes de avaliação cognitiva. A abordagem mais próxima de uma sistematização é a revisada por Judd e cols. (2009), que relata a importância da International Test Comission como órgão regulamentador e guia do uso de testes adaptados e/ou traduzidos (International Test Commission, 2002).

Portanto, uma sistematização mais ampla e diretiva quanto ao processo de adaptação propriamente dito torna-se essencial. Nessa sistematização, a base teórica centra-se na interface entre a neuropsicologia e a psicologia cognitiva, aliadas à neuropsicolinguística e à psicometria e avaliação psicológica. A adaptação de testes neuropsicológicos pode ser conceituada como um processo de construção e de uso de testes que re- 
querem uma sensibilidade linguístico-cultural a ser garantida por sua adequação à língua das pessoas que serão testadas, na medida em que serão utilizados com falantes não nativos da língua e da cultura nas quais o instrumento foi primeiramente desenvolvido (van de Vijver, 2002).

A partir desse pressuposto, com o presente artigo pretende-se responder à seguinte questão: quais são os procedimentos gerais e específicos que devem compor um processo cuidadoso de adaptação de instrumentos neuropsicológicos com estímulos verbais, com ênfase na adaptação semântica ou neuropsicolinguística? Tal questionamento será respondido com base em reflexões teórico-metodológicas acerca das particularidades do processo de geração de uma versão estrangeira de um dado instrumento em uma versão para o Português Brasileiro, transcendendo à mera tradição de traduzir estímulos linguísticos de um país para outro. Alicerçado nesta reflexão, propõe-se um fluxograma de procedimentos de adaptação neuropsicolinguística, que pode contribuir para embasar processos de adaptação de instrumentos neuropsicológicos internacionais com estímulos verbais no contexto brasileiro.

\section{Estudos de adaptação no contexto nacional e internacional}

De acordo com uma breve revisão não sistemática na literatura nacional e internacional, observa-se um crescente esforço na adaptação de instrumentos neuropsicológicos verbais e não verbais no Brasil e demais países. Como exemplo, pode-se citar, em relação à memória verbal episódica, o Teste de Aprendizagem Auditivo-Verbal de Rey, adaptado por Malloy-Diniz, Cruz, Torres e Consenza (2003). Além deste, há a adaptação de instrumentos como o Teste do Relógio (Atalaia-Silva \& Lourenço, 2008) e o Iowa Gambling Task (adaptado por Malloy-Diniz e cols., 2008 e por Schneider \& Parente, 2006), que se destinam a avaliar praxias e componentes de funções executivas, respectivamente.

No âmbito da linguagem, como ilustração do processo de adaptação, mencionam-se a Bateria Montreal de Avaliação da Comunicação adaptada por Fonseca, Parente, Côté e Joanette (2007) e o Protocolo MontrealToulouse de Exame da Afasia MT-86 (Nespoulous, Joanette, \& Lecours, 1986), que está em processo de adaptação para o Português Brasileiro (Soares e cols., 2008), em que os autores destacam a importância da observação de critérios psicolinguísticos e verificação de parâmetros neuropsicométricos. Com o intuito de estimular ações clínico-científicas que melhorem cada vez mais a qualidade de medidas de desempenho linguístico, na literatura nacional estudos com diferentes protocolos de avaliação de linguagem destacam a preocupação com a padronização dos instrumentos (Mansur, Radanovic, Araújo, Taquemori, \& Greco, 2006; Radanovic \& Mansur, 2002; Radanovic e cols., 2004; Soares \& Ortiz, 2008, 2009). São necessários, para tanto, mais estudos teóricos e empíricos que efetivem tais ações.

$\mathrm{Na}$ adaptação de testes não verbais, é comum o emprego dos seguintes passos para adaptação: tradução das instruções originais, retro-tradução (que consiste na tradução para a língua original novamente, por um especialista nas duas línguas em questão), e, em alguns casos, as traduções e retro-traduções são julgadas por uma terceira pessoa proficiente na língua original do instrumento (Atalaia-Silva \& Lourenço, 2008; Malloy-Diniz e cols., 2008; Pérez-Martinez, Baztán, González-Becerra, \& Socorro, 2005; Periáñez \& Barceló, 2001). A adaptação do Iowa Gambling Task, por exemplo, contou com a tradução do instrumento realizada por um primeiro profissional proficiente em inglês. Em seguida, o texto traduzido para o português passou por uma retro-tradução realizada por um segundo profissional proficiente em inglês. Finalmente, as duas versões foram julgadas por dois outros pesquisadores também fluentes em inglês. O processo de adaptação do Teste do Relógio para uso no Brasil por Atalaia-Silva e Lourenço (2008) apresentou características semelhantes, como a tradução das instruções (realizada por um profissional proficiente na língua original do instrumento), julgada em seguida por um outro profissional também fluente.

Em relação à adaptação de instrumentos verbais, etapas de tradução, retro-tradução, julgamento por especialistas (profissionais experts da área de aplicação do instrumento), aplicação de estudos piloto também são realizadas (Carvalho \& Caramelli, 2007; Osório, Crippa, \& Loureiro, 2008). Porém, percebe-se uma preocupação maior no que se refere aos estímulos que possam não corresponder à realidade sóciolinguístico-cultural do país ao qual o teste esteja sendo adaptado. Dessa forma, aspectos psicolinguísticos inerentes à língua tendem a ser considerados com maior ênfase. Por exemplo, no estudo de Fonseca e cols. (2007), o uso de metáforas foi verificado em coleta coletiva durante o processo de adaptação da Bateria MAC, com o intuito de averiguar se essas faziam parte do contexto cultural em que o instrumento seria empregado. 
Rochele Paz Fonseca, Fabíola Schwengber Casarin, Camila Rosa de Oliveira, Gigiane Gindri, Ellen Cristina Siqueira Soares Ishigaki, Karin Zazo Ortiz, Maria Alice de Mattos Pimenta Parente, \& Lilian Cristine Scherer

Um estudo conduzido por Acevedo e cols. (2009) propõe guias gerais no processo de adaptação de instrumentos neuropsicológicos verbais e não verbais à língua espanhola (Boston Naming Test, Trail Making Tes, Logical Memory, Category Fluency Test, Digit Span, Mini Mental e Digit Symbol), alertando para a necessidade da distinção entre tradução literal e adaptação de um instrumento, visto que, no primeiro, parte-se de uma tradução direta, o que resulta em expressões não naturalizadas, comprometendo a mensuração dos componentes cognitivos/linguísticos alvo. Além disso, ressaltam o uso de equivalentes culturais e linguísticos dos estímulos dos testes da língua original para a língua espanhola, assim como o emprego de instruções adaptadas, isto é, as instruções propostas pelo protocolo original são modificadas apenas para torná-las mais compreensíveis, principalmente para pessoas de baixa escolaridade, sem alterar a essência do que é avaliado.

Mais especificamente em investigações transculturais de instrumentos verbais, nos estudos para a adaptação do Teste de Boston para o Diagnóstico das Afasias ao Brasil, as autoras demonstram sua preocupação com a adaptação das estruturas linguísticas do teste, uma vez que o Português Brasileiro não segue a mesma estrutura da língua inglesa (Mansur e cols., 2006; Radanovic \& Mansur, 2002). Após a tradução, em alguns subtestes foram realizadas substituições dos estímulos que apresentavam forte relação com a cultura anglo-saxônica. Além disso, também foram substituídos aqueles que não ficaram adequados para uma determinada tarefa, devido a mudanças na constituição silábica e fonêmica, sendo, então, considerados os critérios utilizados pelos autores da versão original (Radanovic \& Mansur, 2002).

Outro instrumento que também tem sido usualmente referido na literatura nacional (Lecours, Parente, Feijó, \& Maia, 2001; Ortiz, Ferreira \& Bento, 2006) é o Protocolo Montreal-Toulouse de Exame Linguístico da Afasia MT-86 (Protocole Montreal-Toulouse d'examen linguistique de l'aphasie MT-86, elaborado por Nespoulous e cols., 1986) em uma parceria canadense e francesa. O MT-86 é composto por provas capazes de caracterizar a produção e a compreensão oral e gráfica, além da repetição e da fluência verbal. Este teste foi desenvolvido para verificar as alterações de linguagem encontradas em indivíduos afásicos, ou seja, com dificuldades adquiridas de linguagem.

Mesmo sendo um teste com estímulos linguísticos e pictográficos próximos a situações cotidianas da população brasileira, há instruções e estímulos, tanto verbais quanto não verbais, de difícil adequação cultural no referido instrumento (Ortiz, Osborn, \& Chiari, 1993; Soares, 2007). Deve-se salientar que esta ferramenta foi preliminarmente adaptada para o Brasil na década de 1980, sem ter sido publicada, e que atualmente estes estímulos devem ser estudados para que o teste possa ser composto de estímulos mais sensíveis e específicos para diferenciar indivíduos normais de afásicos.

Os autores brasileiros (Parente, Ortiz, SoaresIshigaki, Scherer, Fonseca, Joanette, Lecours, \& Nespoulous, no prelo) realizaram a adaptação do Protocolo Montreal-Toulouse de Exame Lingiístico da Afasia MT-86, que será denominado Bateria MontrealToulouse de Avaliação da Linguagem - Bateria MTL - Brasil. Para a adaptação deste instrumento foi efetuada a tradução e retro- tradução da versão francófona do protocolo, além da utilização da versão do instrumento já adaptado no Brasil na década de oitenta e dos pressupostos neuropsicolinguísticos, experimentais e psicométricos sugeridos na literatura (Lezak, Howienson, \& Loring, 2004; Strauss e cols., 2006). Até mesmo estímulos não verbais demandam adequações culturais por sua relação próxima às representações linguísticas. Para a adaptação dos estímulos pictográficos buscou-se a proximidade com a realidade brasileira, como no caso de uma figura de um homem escalando uma montanha que foi substituída por um homem pintando uma parede. Além disso, todos os estímulos pictográficos foram redesenhados para reduzir as possibilidades de dificuldade de nomeação por questões visuais.

Os estímulos verbais de palavras seguiram critérios de frequência na língua segundo parâmetros brasileiros (Berber Sardinha, 2004), além de critérios de categorias lexicais e semânticas, regularidade, imageabilidade, extensão, operatividade e familiaridade dos estímulos (Raymer \& Rothi, 2001). Os estímulos verbais de frases seguiram o critério de proximidade com a realidade brasileira, além da manutenção das estruturas sintáticas. As estruturas textuais foram adaptadas seguindo o critério de proximidade com a realidade brasileira, a manutenção das estruturas textuais, sintáticas e de sentido, do número de palavras e de proposições.

Além das etapas citadas acima, existe a necessidade de implementação de outros estágios com o intuito de deixar o trabalho de adaptação ainda mais rigoroso e sistematizado. Entre eles, podem-se citar a realização de coletas piloto durante o processo de obtenção de diferentes versões adaptadas, a averiguação da compreensão dos participantes às instruções e de exami-

Interação em Psicologia, Curitiba, 2011, 15(n. especial), p. 59-69 
nadores às informações dos manuais de aplicação e de pontuação, julgamento por experts, entre outros, os quais nem sempre fazem parte dos procedimentos relatados em todos os estudos.

$\mathrm{Na}$ adaptação de instrumentos neuropsicológicos para a pesquisa e a clínica, com estímulos de ordem verbal, dois grandes processos de adaptação devem ser conduzidos: (1) adaptação neuropsicolinguística e (2) adaptação neuropsicométrica. O primeiro processo, também conhecido como adaptação linguística ou semântica, diz respeito à necessidade de condução de um conjunto de procedimentos de análise da influência de critérios psicolinguísticos na neurocognição, possibilitando o controle ou a manipulação de cada critério quando forem necessários. Vem sendo denominada como adaptação semântica, nomenclatura que implica um conjunto de procedimentos rigorosos de adaptação linguística e cultural de instrumentos (Chan, Shum, \& Cheung, 2003; Fonseca e cols., 2007). A adaptação neuropsicométrica trata do conjunto de procedimentos empíricos de adequação dos instrumentos aos parâmetros psicométricos de fidedignidade, validade e de obtenção de normas, com seleção de técnicas ajustadas às demandas e às particularidades das ferramentas de exame do desempenho neuropsicológico.

\section{Contribuições da neuropsicolinguística}

A linguística viu emergir nas últimas décadas um novo e promissor campo de investigação, a neuropsicolinguística. Tem como objeto de estudo a compreensão e a produção da linguagem e sua relação com os processos cognitivos desenvolvidos no cérebro, em populações de diferentes faixas etárias, acometidas ou não de lesão cerebral, com um funcionamento linguístico normal ou patológico, mono-, bi- ou multilíngues, entre outros objetos de estudo sobre a linguagem à luz de uma abordagem neuropsicológica.

A elaboração e adaptação de testes neuropsicológi$\cos$ que avaliam a linguagem ou outras habilidades cognitivas por intermédio de tarefas verbais não encontram respaldo suficiente tanto na literatura nacional quanto na estrangeira no que tange aos critérios neuropsicolinguísticos a serem observados. Em outras palavras, pesquisadores que queiram elaborar uma bateria de testes ou tarefas linguísticas clínicas dificilmente conseguem evidências sobre os critérios neuropsicolinguísticos a serem adotados. Enfim, vários critérios neuropsicolinguísticos precisam ser considerados a fim de que se possa analisar, de modo seguro e dissociado, o processamento dos diferentes componentes linguísticos que podem ser afetados, por exemplo, por acometimento neurológico: os componentes fonológicos, morfológicos, sintáticos, semânticos, discursivos e pragmáticos.

$\mathrm{O}$ cuidado com a linguagem deve ser estendido desde a elaboração das instruções aos participantes e dos manuais de aplicação até os estímulos linguísticos per se. A atenção aos estímulos é fundamental, pois são eles que avaliarão a própria linguagem e outras funções cognitivas e seus respectivos correlatos neurobiológicos. Por exemplo, por meio da linguagem, pode-se avaliar memória (estímulos verbais em uma lista a ser armazenada e posteriormente evocada), atenção (estímulos verbais a serem controlados mentalmente em uma tarefa de evocação das letras do alfabeto em ordem inversa), funções executivas (tarefas de planejamento verbal e de fluência verbal), dentre outras funções cognitivas.

As tarefas linguísticas podem tanto avaliar a produção (oral ou escrita) como a compreensão de linguagem (falada ou lida), em vários níveis - palavras, frases ou discurso, incluindo neste, questões pragmáticas, como as que englobam a ironia, a inferenciação, a teoria da mente, a capacidade de compreender a ideia central, dentre outras. A complexidade do discurso pode ser explicada pelo fato de um texto lido ou ouvido somente ter sentido quando o leitor/ouvinte com ele interagir, atribuir a ele um sentido a partir das pistas dadas pelo texto e relacioná-lo ao contexto da produção. $\mathrm{O}$ discurso tem sido o mais negligenciado nos testes que avaliam a capacidade linguística, possivelmente pelo fato de ser o nível mais complexo, de mais longa avaliação, ou ainda por uma percepção errônea ou limitada do que venha a ser linguagem, por parte dos que a limitam ao nível da palavra ou da sentença, desconsiderando sua importante função em seus contextos reais de uso, em que o discurso predomina. Os critérios psicolingüísticos a serem observados na construção de tarefas para avaliação do processamento discursivo podem ter um caráter mais lingüístico, ligado à estrutura de superfície do texto, ou mais pragmático-discursivo, ligado a aspectos que demandam o processamento de informação nem sempre explicitamente dada (Dell'Isola, 2001). Dentre os critérios ligados à superfície do texto, destacam-se a extensão do texto, o número de palavras e de proposições (Van Dijk, 1997), a complexidade das estruturas sintáticas e semânticas das orações (Friederici \& Weissenborn, 2007), os aspectos estruturais relacionados à coesão textual (Dascal, 2006), a coerência textual (Coscarelli, 2003; Kintsch, 1998), dentre ou- 
Rochele Paz Fonseca, Fabíola Schwengber Casarin, Camila Rosa de Oliveira, Gigiane Gindri, Ellen Cristina Siqueira Soares Ishigaki, Karin Zazo Ortiz, Maria Alice de Mattos Pimenta Parente, $\&$ Lilian Cristine Scherer

tros critérios. Dentre os fatores pragmático-discursivos, destacam-se o grau de informações implícitas, a exigência de conhecimento prévio devido à temática do texto (Kleiman, 2004; Kock, 2001), a ocorrência ou não de ironia, dentre outras questões.

No que tange ao nível da frase, os mesmos critérios assinalados como sendo importantes na adaptação ou criação de textos para avaliar a linguagem devem ser observados, aliados a outros, como a plausibilidade (sentenças podem ser verdadeiras ou falsas, bizarras, como em "O menino floresce a enchente") e o grau de informações implícitas e explícitas contidas na frase (quanto mais explícita a informação, mais facilitado é o processamento).

Finalmente, no nível da palavra, alguns dos critérios a serem levados em consideração na adaptação de testes neuropsicológicos são: a frequência (Berber Sardinha, Moreira Filho, \& Alambert, 2007), a familiaridade, o grau de imageabilidade, o número de sílabas; a tonicidade, a prototipicalidade e as noções relacionadas, como gradiência e indeterminação, ligadas à categorização linguística (Aimé, Furst, Kuntz, \& Trichet, 2008), a especificidade quanto ao uso entre os diferentes sexos; o significado conotativo ou denotativo e a convencionalidade (Tagnin, 1989, 2005).

\section{Contribuições da psicometria}

A psicometria contribui com seus pressupostos para a verificação da fidedignidade, da validação (evidências de validade de conteúdo, de construto e de critério) e da normatização de testes neuropsicológicos. Para uma revisão sobre definições constitutivas e operacionais destes parâmetros, recomenda-se a consulta a obras clássicas e renomadas, nacionais e internacionais, de psicometria (por exemplo, Anastasi \& Urbina, 2000; Pasquali, 1999, 2001, 2003).

$\mathrm{Na}$ avaliação neuropsicológica, a psicometria tem um papel diferenciado da contribuição dada à mensuração psicológica clássica, uma vez que o desempenho observado na primeira é necessariamente relacionado com o funcionamento cognitivo geral e/ou com o do sistema nervoso. $\mathrm{O}$ instrumento empregado deve ser combinado, ainda, com os resultados de outros testes (Benedet, 2002). Russel, Russel e Hill (2005) concordam que as tarefas das baterias neuropsicológicas são combinadas ou comparadas para a interpretação das funções cognitivas.

Gauer, Gomes e Haase (2010) afirmam que as questões psicométricas em baterias neuropsicológicas são complexas e comentam sobre três tipos peculiares de validade: ecológica, de localização e diagnóstica. A validade ecológica prima pela capacidade de os instrumentos predizerem o funcionamento na realização de tarefas reais, considerando as demandas do contexto clínico e do cotidiano do paciente, enquanto que a validade de localização relaciona-se com o desempenho obtido e seus correlatos neuroanatômicos. Não menos importante, a validade diagnóstica diz respeito à contribuição da medida neuropsicológica no diagnóstico neurológico. Entretanto, são diversos os tipos de validade cujas evidências podem ser buscadas. Pasquali (2007) revisa 31 validades e reflete sobre a conusão terminológica que cerca este parâmetro psicométrico. Além da fidedignidade, da validade e da normatização, quanto ao interesse neuropsicológico clínico, dois critérios são essenciais de serem estudados no processo de adaptação neuropsicométrica de um instrumento de avaliação cognitiva: a sensibilidade e a especificidade, medidas de acurácia diagnóstica (para uma revisão consultar Lezak e cols., 2004; Strauss e cols., 2006).

De modo mais específico e relacionado à neuropsicologia, no presente artigo defende-se o uso e a valorização do termo neuropsicometria. Desde a seleção até a condução e a interpretação de estudos teóricoempíricos de adaptação psicométrica, é fundamental uma reflexão sobre características específicas da avaliação neuropsicológica, não necessariamente presentes na avaliação psicológica como um todo. Pawlowski, Trentini e Bandeira (2007) abordam aspectos desta análise reflexiva na adaptação psicométrica de instrumentos neuropsicológicos. Figueroa e Schmidt (2008) dissertam, ainda, sobre as relações de semelhança e de distinção entre avaliação psicológica e neuropsicológica.

No contexto nacional, percebem-se esforços de pesquisadores em incluir em seus estudos de construção ou de adaptação procedimentos psicométricos (Fonseca e cols., 2007; Ortiz e cols., 2008; Pawlowski e cols., 2008). No entanto, observa-se que, no processo de normatização, variáveis sociodemográficas que afetam o desempenho cognitivo (como idade, anos de estudo, frequência de hábitos de leitura e escrita, sexo) algumas vezes ainda são negligenciadas ou insuficientemente consideradas ao se definirem os grupos normativos.

No intuito de refletir sobre a adaptação de instrumentos neuropsicológicos, haja vista a lacuna existente nesta temática, na próxima seção traz-se uma proposta de fluxograma do processo de adaptação neuropsicolinguística, que deve ser sucedido pelo de 
adaptação neuropsicométrica. As etapas propostas procuram enfatizar as particularidades da adaptação de testes com estímulos verbais, ferramentas muito utilizadas na prática neuropsicológica brasileira.

\section{Proposta de um fluxograma de adaptação neuropsicolingüística}

O fluxograma proposto tem como objetivo sugerir as etapas que podem ser seguidas no processo de adaptação de instrumentos neuropsicológicos verbais para a realidade brasileira (Figura 1). Pode-se dizer que existem quatro etapas fundamentais nesse processo: tradução, análise de juízes não especialistas (ou seja, indivíduos provenientes de diferentes grupos sociais e profissionais, porém com mesma faixa etária e nível de escolaridade dos da população a ser dirigida no teste), análise de juízes especialistas e estudo piloto. Cabe ressaltar que, após cada etapa, a análise dos autores do teste é de extrema importância, assim como o contato constante entre coordenadores da adaptação e autores do instrumento original.

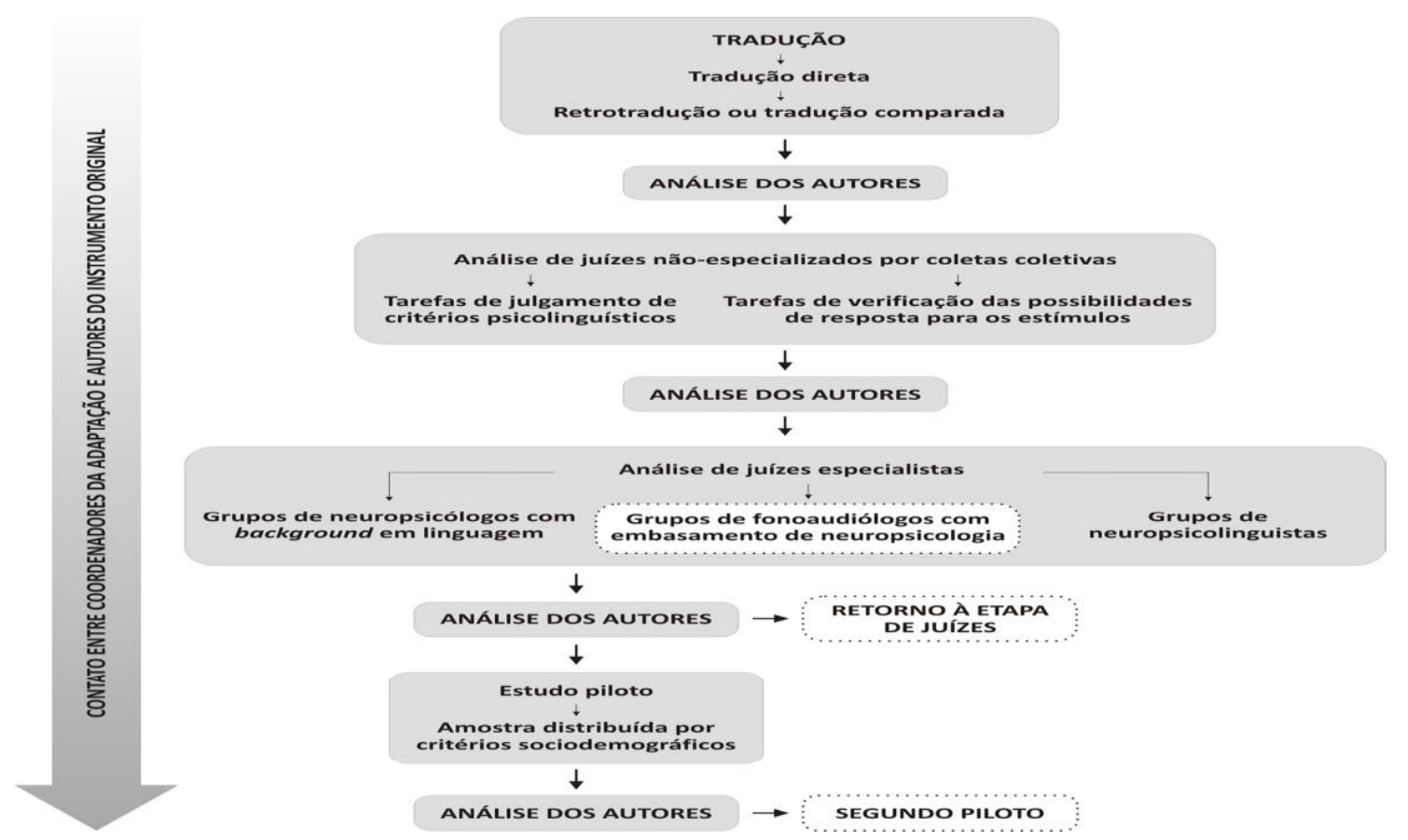

Figura 1. Fluxograma de adaptação de instrumentos neuropsicológicos com estímulos verbais.

A primeira etapa corresponde à tradução do teste original por dois tradutores independentes. Após, realiza-se uma retro-tradução (ou tradução reversa) ou tradução comparada, em que um terceiro tradutor fluente nas duas línguas julga os melhores termos para fazer parte da bateria. Para completar a versão traduzida, ajustes terminológicos são, ainda, promovidos pelos autores, salientando-se que esse procedimento deve ser considerado mais importante do que uma simples tradução, pois os termos adaptados ao contexto sócio-linguístico brasileiro não podem desconsiderar os objetivos originais de cada tarefa.

Nessa fase, ocorre a adaptação semântica, que compreende a equivalência de significado entre a língua da qual o teste é originário para aquela na qual será adaptado, isto é, mantém-se a clareza de conteúdo e os critérios de familiaridade e psicolinguísticos, observando-se diferentes formas de expressões nacionais (Anastasi \& Urbina, 2000; Pasquali, 2001). Essa equivalência pode ser obtida pelo julgamento dos juízes especialistas e não especialistas, e, finalmente, os autores fazem o consenso.

A segunda etapa caracteriza-se pela análise de juízes não especialistas por meio de coletas coletivas. Os juízes não especialistas são pessoas da mesma faixa etária e de escolaridade da população a ser investigada pelo instrumento. Esses juízes analisarão as tarefas fazendo um julgamento de critérios psicolinguísticos, isto é, julgarão o quão familiar é o estímulo selecionado para determinada tarefa, segundo a experiência 
Rochele Paz Fonseca, Fabíola Schwengber Casarin, Camila Rosa de Oliveira, Gigiane Gindri, Ellen Cristina Siqueira Soares Ishigaki, Karin Zazo Ortiz, Maria Alice de Mattos Pimenta Parente, \& Lilian Cristine Scherer

linguiística do cotidiano deles. Além desta consulta a representantes da população em geral quanto a critérios neuropsicolinguísticos, nesta coleta coletiva, fazse a verificação das possibilidades de respostas dos estímulos (procedimento em que escreverão o que significa a palavra ou frase do estímulo apresentado). Após esse julgamento, os autores selecionam os melhores estímulos para a bateria de avaliação, aqueles que não são tão fáceis e nem tão difíceis. Não há uma quantidade de juízes não especialistas preestabelecida na literatura, nem cálculos amostrais, mas preconizase distribuição por áreas e por regiões geográficas do país. Recomenda-se ao redor de 10 a 20 não especialistas por critérios sociodemográficos a serem considerados na futura normatização. Assim, os estímulos selecionados serão condizentes com o conhecimento da população para a qual o instrumento se destinará.

A próxima etapa é a análise por juízes especialistas que, no caso da adaptação de um instrumento verbal, são neuropsicólogos com background em linguagem, um grupo de fonoaudiólogos com embasamento da neuropsicologia (caso o teste avalie a própria linguagem) e um grupo de neuropsicolinguistas e linguistas. A função desses profissionais é sugerir modificações tanto na aplicação quanto nos estímulos que compõem as tarefas. Conforme as sugestões dadas, os autores analisam novamente o instrumento e, caso seja necessário, após as modificações, há um retorno aos juízes especialistas. Recomenda-se a quantidade de 12 juízes, sendo a maioria de neuropsicológos com conhecimento do processamento linguístico.

Em muitos países, há bancos de dados e normas sobre critérios linguísticos, como o grau de concretude. No caso do Brasil, em que muitos dos bancos não estão disponíveis por não terem ainda sido desenvolvidos, tornam-se necessárias estas etapas de julgamento acima descritas.

Por fim, a última etapa é o estudo piloto com uma amostra distribuída por critérios sociodemográficos. A partir desse procedimento, as dificuldades encontradas pelos participantes do estudo podem ser posteriormente solucionadas, assim como, as sugestões por eles promovidas poderão ser consideradas para o aprimoramento do instrumento adaptado. Os autores analisam mais uma vez as críticas e sugestões e sendo necessário um segundo piloto é promovido. Não há na literatura, até onde se sabe, uma indicação do tamanho amostral de um piloto. Sugere-se pela experiência com os instrumentos adaptados até então que sejam incluídos dois indivíduos saudáveis por futuro grupo normativo (por exemplo, duas pessoas com 20 a 30 anos de idade e 5 a 8 de escolaridade), além de pelo menos dois pacientes com o diagnóstico do quadro para o qual o instrumento foi desenvolvido.

\section{CONSIDERAÇÕES FINAIS}

A proposta de etapas de adaptação de instrumentos verbais apresentada neste artigo é um primeiro passo para a reflexão sobre uma possível sistematização do processo de adaptação neuropsicolinguística, com o intuito de possibilitar adequação dessas ferramentas visando a sua inserção sócio-linguístico-cutural. Alguns parâmetros psicométricos como a validação e a normatização de um instrumento são frequentemente descritos tanto na construção quanto na adaptação de ferramentas psicológicas e neuropsicológicas. Em contrapartida, a preocupação com aspectos psicolinguísticos e com sua influência no processamento neuropsicológico ainda está despontando. Particularmente, no que tange aos estímulos verbais, destaca-se a necessidade de um maior controle dos critérios neuropsicolinguísticos, pois a linguagem é veículo de mensuração de outras funções cognitivas ou a ferramenta de avaliação de si própria.

Essas reflexões sobre a importância de uma rigorosa adaptação ainda precisam ser mais exploradas e divulgadas, alertando-se os pesquisadores para o fato de que essa preocupação deva extrapolar os instrumentos verbais. Na avaliação neuropsicológica é preciso estar atento para que os instrumentos adaptados mantenham os objetivos propostos quando da sua construção e que as explicações dadas ao examinando estejam claras quanto à forma e ao conteúdo, também no emprego de testes não verbais. A compreensão da instrução é essencial, independentemente da modalidade do instrumento, para que os processos cognitivos sejam mensurados conforme o previsto.

As contribuições da neuropsicometria associadas à adaptação neuropsicolinguística que a antecede, assim como sua interdependência devem ser mais bem investigadas para que instrumentos normatizados e padronizados possam ser cada vez mais acurados na prática neuropsicológica. Esta proposta de seguir um fluxograma com observação flexível das etapas de adaptação de instrumentos neuropsicológicos que utilizem estímulos verbais pode contribuir também para uma reflexão sobre a construção de instrumentos neuropsicológicos padronizados. Finalmente, ressaltase que uma elaboração criteriosa da tarefa experimental com estímulo linguístico é fundamental, tanto nos estudos comportamentais quanto nos de neuroimagem 
funcional, para que o estímulo não contamine os dados e leve a conclusões equivocadas.

\section{REFERÊNCIAS}

Anastasi, A., \& Urbina, S. (2000). Testagem psicológica (M. A. V. Veronese, Trad.). Porto Alegre: Artmed.

Acevedo, A., Krueger, K. R., Navarro, E., Ortiz, F., Manly, J. J., Padilla-Vélez, M. M., Weintraub, S., López, O. L., \& Mungas, D. (2009). The Spanish translation and adaptation of the Uniform Data Set of the National Institute on Aging Alzheimer's Disease Centers. Alzheimer Disease and Association Disorders, 23, 102-109.

Aimé, X., Furst, F., Kuntz, P., \& Trichet, F. (2008). Conceptual and lexical prototypicality gradients dedicated to ontology personalization. Berlim: Springer Verlag/Heidelberg.

Atalaia-Silva, K. C., \& Lourenço, R. A. (2008). Tradução, adaptação e validação de construto do Teste do Relógio aplicado entre idosos no Brasil. Revista de Saúde Pública, 42, 930-937.

Benedet, M. J. (2002). Neuropsicología cognitiva: Aplicaciones a la clínica y a la investigación. Madri: Instituto de Migraciones y Servicios Sociales.

Berber Sardinha, T. (2004). Lingüística de corpus. Barueri: Manole.

Berber Sardinha, T., Moreira Filho, J. L., \& Alambert, E. (2007), Banco de português. São Paulo: CEPRIL.

Carvalho, V.A., \& Caramelli, P. (2007). Brazilian adaptation of the Addenbrooke's Cognitive Examination-Revised (ACE-R). Dementia \& Neuropsychologia, 2, 212-216.

Casillas, A., \& Robbins, S. B. (2005). Test adaptation and crosscultural assessment from a business perspective: Issues and recommendations. International Journal of Testing, 5, 5-21.

Chan, A. S., Shum, D., \& Cheung, R. W. Y. (2003). Recent development of cognitive and neuropsychological assessment in Asian countries. Psychological Assessment, 15, 257-267

Cronbach, L. J. (1996). Fundamentos da testagem psicológica (C. A. S. Neto \& M. A. V. Veronese, Trad.). Porto Alegre: Artes Médicas.

Coscarelli, C. V. (2003). Inferência: Afinal, o que é isso? Belo Horizonte: FALE/ UFMG.

Cunha, J. A. (2000). Psicodiagnóstico - V. Porto Alegre: Artmed.

Dell'Isola, R. L. P. (2001). Leitura: Inferências e contexto sociocultural. Belo Horizonte: Formato.

Figueroa, N. L., \& Schmidt, V. (2008). Reflexiones acerca de la evaluación psicológica y neuropsicológica. Revista Argentina de Neuropsicología, 12, 21-28.

Fonseca, R. P., Parente, M. A. P., Côté, H., \& Joanette, Y. (2007). Processo de adaptação da Bateria Montreal de Avaliação da Comunicação - Bateria MAC - ao português brasileiro. Psicologia: Reflexão \& Crítica, 20, 259-267.

Friederici, A. D., \& Weissenborn, J. (2007). Mapping sentence form onto meaning: The syntax-semantic interface. Brain Research, 1146, 50-58.

Gauer, G., Gomes, C. M. A., \& Haase, V. G. (2010). Neuropsicometria: Modelo clássico e análise de Rasch. Em L. F. MalloyDiniz, D. Fuentes, P. Mattos, N. Abreu e cols. (Orgs.), Avaliação neuropsicológica (pp. 22-30). Artmed: Porto Alegre.
Guillemin, F. (1995). Cross-cultural adaptation and validation of health status measures. Scandinavian Journal of Rheumatology, 24, 61-63.

Hebben, N., \& Milberg, W. (2002). Essentials of neuropsychological assessment. New York: John Wiley \& Sons.

International Test Commission. (2002). International Test Commission test adaptation guidelines. Retirado em 14 de janeiro de 2004, de www.intestcom.org

Judd, T., Capetillob, D., Carrion-Baralt, J. Mármold, L. M., Miguel-Montes, L. S., Navarrete, M. G., Puente, A. E., Romero, H. R., Valdés, J., \& the NAN Policy and Planning Committee (2009). Evaluation of hispanics: A National Academy of Neuropsychology education paper. Archives of Clinical Neuropsychology, 24, 127-135.

Kintsch, W. (1998). Comprehension: A paradigm for cognition. New York: Cambridge University Press.

Kleiman, A. (2004). Oficina de leitura. Campinas: Pontes.

Kock, I. (2001). O texto e a construção dos sentidos. São Paulo: Contexto.

Kolb, B., \& Whishaw, I. Q. (2006). Neuropsicología humana (S. Cwi, D. Klajan, A. Latrónico, U. Patrone, J. Oxemberg, S. Rondinone \& J. Tzal, Trad.). Buenos Aires: Panamericana.

Labos, E., Slachevsky, A., Fuentes, P., \& Manes, F. (2008). Tratado de neuropsicología clínica. Buenos Aires: Akadia.

Lecours, A. R., Parente, M. A. M. P., Feijó, A. V., \& Maia, A. (2001). Anartria pura: Estudo de dois casos. Psicologia: Reflexão e Crítica, 14, 367-377.

Lezak, M. D., Howienson, D. B., \& Loring, D. W. (2004). Neuropsychological assessment. New York: Oxford University Press.

Loschiavo, F., Freitas, J. A. C., Malloy-Diniz, L., \& Cosensa, R. M. (2008). Adaptação brasileira da bateria de Strub-Black. Em A. F. Veloso, L. I. Z. Mendonça, K. Z. Ortiz, D. Fuentes \& D. A. Azambuja (Orgs.), Avaliação neuropsicológica no Brasil: Panorama interdisciplinar dos instrumentos em estudo (pp. 5871). São Paulo: SBNp.

Malloy-Diniz, L. F., Lasmar, V. A. P., Gazinelli, L. S. R., Fuentes, D., \& Salgado, J. V. (2007). The Rey Auditory-Verbal Learning Test: Applicability for the Brazilian elderly population. $R e$ vista Brasileira de Psiquiatria, 29, 324-329.

Malloy-Diniz L. F., Cruz, M. F., Torres, V., \& Cosenza, R. (2003). O Teste de Aprendizagem Auditivo-Verbal de Rey: Normas para uma população brasileira. Revista Brasileira de Neurolologia, 36, 79-83.

Malloy-Diniz, L. F., Leite, W. B., Moraes, P. H. P., Correa, H., Bechara, A., \& O Fuentes, D. (2008). Brazilian Portuguese version of the Iowa Gambling Task: Transcultural adaptation and discriminant validity. Revista Brasileira de Psiquiatria, 30, 144-148.

Mansur, L. L., Radanovic, M., Araújo, G. C., Taquemori, L.Y., \& Greco, L. L. (2006). Teste de nomeação de Boston: Desempenho de uma população de São Paulo. Pró-Fono, 18, 13-20.

Nascimento, E., \& Figueiredo, V. L. M. (2002). WISC-III e WAIS-III: Alterações nas versões originais americanas decorrentes das adaptações para uso no Brasil. Psicologia: Reflexão e Crítica, 15, 606-612.

Nespoulous, J. L., Joanette, Y., \& Lecours, A. R. (1986). Protocole Montréal-Toulouse d'examen linguistique de l'aphasie (MT-86). Isbergues: Ortho Édition. 
Ortiz, K. Z., Ferreira, C. P., \& Bento, A. C. P. (2006). Aplicação do teste Beta 86 (protocolo MT modificado) em analfabetos. Fono Atual (São Paulo), 35, 65-73.

Ortiz, K. Z., Mendonça, L. I. Z, Foz, A., Santos, C. B., Fuentes, D., \& Azambuja, D. A. (2008). Avaliação neuropsicológica: Panorama interdisciplinar dos estudos de normatização e validação de instrumentos no Brasil. São Paulo: Vetor.

Ortiz, K. Z., Osborn, E., \& Chiari, B. M. (1993). O teste M1Alpha como instrumento de avaliação da afasia. Pró-Fono, 5, 23-29.

Osório, F. L., Crippa, J. A., \& Loureiro, S. R. (2008). Escala para Auto-Avaliação ao Falar em Público (SSPS): Adaptação transcultural e consistência interna da versão brasileira. Revista de Psiquiatria Clínica, 35, 207-211.

Parente, M. A. M. P., Ortiz, K. Z., Soares-Ishigaki, E. C., Scherer, L. C., Fonseca, R. P., Joanette, Y., Lecours, A. R. \& Nespoulous, J. L. (no prelo). Bateria Montreal-Toulouse de Avaliação da Linguagem - Bateria MTL-Brasil. São Paulo: Vetor.

Pasquali, L. (1999). Instrumentos psicológicos: Manual prático de elaboração. Brasília: LabPAM/IBAPP.

Pasquali, L. (2001). Técnicas de exame psicológico - TEP: Мanual. São Paulo: Casa do Psicólogo.

Pasquali, L. (2003). Psicometria: Teoria dos testes na psicologia e na educação. Petrópolis: Vozes.

Pasquali, L. (2007). Validade dos testes psicológicos: Será possível reencontrar o caminho? Psicologia: Teoria e Pesquisa, 23, 99-107.

Pawlowski, J., Fonseca, R. P., Salles, J. F., Parente, M. A. M. P., \& Bandeira, D. R. (2008). Evidências de validade do Instrumento de Avaliação Neuropsicológica Breve NEUPSILIN. Arquivos Brasileiros de Psicologia, 60, 101-116.

Pawlowski, J., Trentini, C. M., \& Bandeira, D. R. (2007). Discutindo procedimentos psicométricos a partir da análise de um instumento de avaliação neuropsicológica breve. Psico-USF, 12, 211-219.

Pérez-Arce, P. (1999). The influence of culture on cognition. Archives of Clinical Neuropsychology, 14, 581-592.

Pérez-Martínez, D. A., Baztán, J. J., González-Becerra, M., \& Socorro, A. (2005). Evaluación de la utilidad diagnóstica de una adaptación española del Memory Impairment Screen de Buschke para detectar demencia y deterioro cognitivo. Revista de Neurología, 40, 644-8.

Periáñez, J. A., \& Barceló, F. (2001). Adaptación Madrid del Test de Clasificación de Cartas de Wisconsin: Un estudio comparativo de consistencia interna. Revista de Neurología, 33, 611618.

Radanovic, M., \& Mansur, L. L. (2002). Performance of a Brazilian population sample in the Boston Diagnostic Aphasia Examination: A pilot study. Brazilian Journal of Medical and Biological Research, 35, 305-317.

Radanovic, M. L., Mansur L. L., \& Scaff, M. (2004). Normative data for the Brazilian population in the Boston Diagnostic
Aphasia Examination: Influence of schooling. Brazilian Journal of Medical and Biological Research, 37, 1731-1738.

Raymer, A. M., \& Rothi, L. J. G. (2001). Cognitive approaches to impairments of word comprehension and production. Em R. Chapey (Org.), Language intervention strategies in aphasia and related neurogenic communication disorders (pp. 524550). Philadelphia: Lippincott Williams \& Wilkins.

Schneider, D. G., \& Parente, M. A. M. P. (2006). O desempenho de adultos jovens e idosos na Iowa Gambling Task (IGT): Um estudo sobre a tomada de decisão. Psicologia: Reflexão e Críti$c a, 19,442-450$.

Soares, E. C. S. (2007). Avaliação de linguagem em sujeitos adultos através do teste MT Beta 86 modificado: Estudo piloto e aplicação em sujeitos afásicos. Dissertação de mestrado nãopublicada, Universidade Federal de São Paulo.

Soares, E. C. S., Fonseca, R. F., Scherer, L. C., Parente, M. A. M. P., Ortiz, K. Z., Joanette, Y., \& Nespoulous, J. L. (2008). Protocolo Montreal-Toulouse de Exame Linguístico da Afasia MT-86: Estudos e perspectivas futuras. Em K. Z. Ortiz, L. I. Z. Mendonça, A. Foz, C. B. Santos, D. Fuentes \& D. A. Azambuja (Orgs.), Avaliação neuropsicológica: Panorama interdisciplinar dos estudos de normatização e validação de instrumentos no Brasil (pp. 275-289). São Paulo: Vetor.

Soares, E. C. S., \& Ortiz, K. Z. (2008). Influence of brain lesion and educational background on language tests in aphasic subjects. Dementia \& Neuropsychologia, 4, 321-327.

Soares, E. C. S., \& Ortiz, K. Z. (2009). Influence of schooling on language abilities of adults without linguistic disorders. São Paulo Medical Journal, 127, 134-139.

Spreen, O., \& Strauss, E. (1998). A compendium of neuropsychological tests: Administration, norms and commentary. New York: Oxford University Press.

Strauss, E., Sherman, E. M. S., \& Spreen, O. (2006). A compendium of neuropsychological tests. New York: Oxford University Press.

Strub, R. L., \& Black, F. W. (2000). The mental status examination in neurology. Philapeldhia: FA Davis.

Tagnin, S. E. O. (1989). Expressões idiomáticas e convencionais. São Paulo: Ática.

Tagnin, S. E. O. (2005). O jeito que a gente diz. São Paulo: Disal.

Van de Vijver, F. (2002). Cross-cultural assessment: Value for money? Applied Psychology, 51, 545-566.

Van Dijk, T. A. (1997). The study of discourse. Em T. A. Van Dijk (Org.), Discourse as structure and process: Discourse studies - A multidisciplinary introduction (pp. 1-34). London: SAGE.

Villaseñor, T., Lopez Carlos, E., Curiel, G. R., Medrano, M. A., \& Rodríguez, R. P. (2003). Validación de instrumentos cognitivos en población infantil en niños exicanos: Estudio preliminar. Trabalho apresentado no VIII Congresso da Sociedade Latinoamericana de Neuropsicologia, Montreal, Canadá 


\section{Sobre as autoras:}

Rochele Paz Fonseca - Psicóloga e fonoaudióloga, Mestre e Doutora em Psicologia do Desenvolvimento pela Universidade Federal do Rio Grande do Sul. Professora do Programa de Pós-Graduação em Psicologia da Pontifícia Universidade Católica do Rio Grande do Sul, área de concentração Cognição Humana. Pós-Doutorada em Medicina (Radiologia) pela Universidade Federal do Rio de Janeiro e em Ciências Biomédicas pela Université de Montréal, Canadá.

Fabíola Schwengber Casarin - Doutoranda e Mestre do Programa de Pós-Graduação em Psicologia, área de concentração Cognição Humana (PUCRS).

Camila Rosa de Oliveira - Mestranda do Programa de Pós-Graduação em Psicologia, área de concentração Cognição Humana (PUCRS).

Gigiane Gindri - Doutoranda do Programa de Pós-Graduação em Psicologia, área de concentração Cognição Humana (PUCRS).

Ellen Cristina Siqueira Soares Ishigaki - Doutoranda do Programa de Pós-Graduação em Fonoaudiologia da Universidade Federal de São Paulo (Escola Paulista de Medicina).

Karin Zazo Ortiz - Fonoaudióloga, com especialização, mestrado e doutorado em Distúrbios da Comunicação Humana pela Universidade Federal de São Paulo. Pós-Doutorado em Neurociências pela Universidade Federal de São Paulo (Escola Paulista de Medicina). Professora do Programa de Pós-Graduação em Fonoaudiologia da Universidade Federal de São Paulo (Escola Paulista de Medicina).

Maria Alice de Mattos Pimenta Parente - Fonoaudióloga, Doutora em Psicologia pela Universidade de São Paulo. Professora convidada do Programa de Pós-Graduação em Psicologia da Universidade Federal do Rio Grande do Sul.

Lilian Cristine Scherer - Graduada em Letras, Doutora em Lingüística pela Universidade Federal de Santa Catarina. Professora do Programa de Pós-Graduação em Letras - Linguística (PUCRS). Pós-Doutorado em Ciências Biomédicas (Universidade de Montreal, UdeM, Canadá).

Endereço eletrônico: rochele.fonseca@pucrs.br 